\title{
Low-velocity collisions of chondrules: How a thin dust cover helps enhance the sticking probability
}

\author{
Nina Gunkelmann ${ }^{1}$, Akimasa Kataoka ${ }^{2}$, Cornelis P. Dullemond ${ }^{2}$, and Herbert M. Urbassek ${ }^{3}$ \\ ${ }^{1}$ Institute for Materials Simulation, Department of Materials Science, Friedrich-Alexander University Erlangen-Nürnberg (FAU), \\ Dr.-Mack-Str. 77, 90762 Fürth, Germany \\ 2 Institut für Theoretische Astrophysik, Universität Heidelberg, Albert-Ueberle-Str. 2, 69120 Heidelberg, Germany \\ 3 Physics Department and Research Center OPTIMAS, University Kaiserslautern, Erwin-Schrödinger-Straße, 67663 Kaiserslautern, \\ Germany \\ e-mail: urbassek@rhrk.uni-kl.de
}

Received 29 November 2016 / Accepted 4 February 2017

\begin{abstract}
Aims. The collision of two chondrules covered by a dust shell is investigated using a granular-mechanics algorithm.

Methods. We focus on the specific case of chondrules of radius $25 \mu \mathrm{m}$ covered by dust of $0.76 \mu \mathrm{m}$ radius; the dust shells have thicknesses of up to $5 \mu \mathrm{m}$ and filling factors between 0.08 and 0.21 .

Results. We demonstrate that the bouncing velocity of the two chondrules increases by two orders of magnitude if a dust shell covers the chondrules. The shells become partly destroyed during the collision process, both by sputtering (monomer ejection) and by agglomeration to dust aggregates. Thicker and denser dust shells are more efficient in accommodating the collision energy than thin and porous shells.
\end{abstract}

Key words. planets and satellites: formation - protoplanetary disks - methods: numerical

\section{Introduction}

Collisions between dust particles or dust-covered objects are ubiquitous in an astrophysical environment (Grün 2007). These collisions are of particular importance in protoplanetary disks, where such collisions ultimately lead - by aggregation processes - to the formation of planets and moons (Blum 2010). While many aspects of such collisions have by now been investigated, the role of collisions between mm- to $\mathrm{m}$-sized objects still poses many questions (Birnstiel et al. 2016). On the one hand, collision velocities of such objects with each other and with smaller dust particles may occur with higher relative velocities, since these objects have (partly) decoupled from their gas environment. On the other hand, the heterogeneous structure of such larger objects complicates the analysis.

Chondrules are prominent mm-sized objects. Today they are found as constituents in meteoritic materials. Isotopic dating of these chondrules indicates that they must have been incorporated into the meteorite parent body during the time of the formation of the solar system. In chondritic meteorites, many chondrules are surrounded by so-called rims, which presumably originate from dust covering the chondrules while they were free-floating in the solar nebula (Metzler et al. 1992; Ormel et al. 2008; Cuzzi 2004).

Studies of collisions between dust-covered chondrules are rare. An important characteristics of such collisions is the socalled bouncing (or sticking) velocity $v_{\mathrm{b}}$ : at collision velocities $v<v_{\mathrm{b}}$, the two colliding chondrules stick, while for $v \geq v_{\mathrm{b}}$ they bounce off each other. Beitz et al. (2012) provide a prominent experimental study by colliding $\mathrm{mm}$-sized dust-covered silica spheres, taken as chondrule analogs, at velocities of a few $\mathrm{cm} / \mathrm{s}$ using drop tower experiments. The dust shells had a thickness of $50 \mu \mathrm{m}$ and filling factors of $0.35-0.58$. These authors find that the bouncing velocity is in the range of a few $\mathrm{cm} / \mathrm{s}$; this is far above the bouncing velocity of dust aggregates of the same size, which amounts to $8 \times 10^{-3} \mathrm{~cm} \mathrm{~s}^{-1}$ (Güttler et al. 2010; Zsom et al. 2010; Weidling et al. 2012).

In this Letter we use a granular-mechanics approach to simulate the collision of naked and dust-covered chondrules. Owing to computational limitations, our chondrules are only $25 \mu \mathrm{m}$ in radius; however, since the dust grains measure only $0.76 \mu \mathrm{m}$ in radius, the dust-chondrule contrast is large enough to allow for reliable conclusions as to the influence of a dust shell on the collision dynamics. Our results show that indeed a dust cover leads to a strong increase of the bouncing velocity of chondrules and allows us to analyze the basic mechanism behind this phenomenon.

\section{Method}

We build the chondrules by constructing spherical clusters of radius $R+d$ and cutting out a sphere of radius $R$, where $d$ is the shell thickness ranging from $d=1.5$ to $d=5 \mu \mathrm{m}$ and $R=25 \mu \mathrm{m}$ is the size of the uncovered chondrule. The spherical clusters are created by adding grains at random to a central particle with the constraint of keeping the average filling factor at a specified value; we used this method since the spherical clusters were already available from a previous study (Gunkelmann et al. 2016). A slightly modified algorithm, which constructs fractal agglomerates, has been published by Ringl \& Urbassek (2013). The filling factor of the dust shells ranges from $\phi=0.08$ to $\phi=0.21$. Thus the total number of grains in a shell varies between 520 for 
the thinnest and most porous shell and 5700 for the thickest and densest shell. As the mass ratio of one grain to a chondrule is $2.8 \times 10^{-5}$, the mass ratio of shell to chondrules varies between 1.5 and $16 \%$.

Our dust shell grains consist of $\mathrm{SiO}_{2}$ grains, each with a radius of $R_{\text {grain }}=0.76 \mu \mathrm{m}$; this is a typical value in experiments on the mechanical properties of porous granular aggregates in the astrophysical context (Blum \& Schräpler 2004). The density of the $\mathrm{SiO}_{2}$ grains and the chondrule amounts to $\rho=2 \times 10^{3} \mathrm{~kg} \mathrm{~m}^{-3}$ (Blum \& Schräpler 2004).

The chondrules collide centrally with relative velocities $v$ varying between 0.05 and $1 \mathrm{~m} \mathrm{~s}^{-1}$. Such velocities are typical of relative velocities in the mid-planes of protoplanetary disks (Birnstiel et al. 2016). In order to gather some statistics, for each collision three individual impacts have been simulated; these impacts differ from each other by an arbitrary rotation of the chondrules before collision. The results shown are averages over these three impacts.

The simulations are performed under periodic boundary conditions. We use a cuboidal simulation box with a length of $2100 \mu \mathrm{m}$ in collision direction and a lateral width of $620 \mu \mathrm{m}$. The large enough box size is chosen to minimize possible boundary condition effects.

The details of our simulation method have been published by Ringl \& Urbassek (2012). The algorithm is implemented in the open-source code LIGGGHTS (Kloss et al. 2012). In the following we only briefly describe our method.

Because of the small grain size appropriate to cosmic dust particles, an accurate description of intergranular adhesion as well as of friction forces (Dominik \& Tielens 1997; Wada et al. 2007) is indispensable.

As is common in granular mechanics, we define the overlap of two grains of radii $R_{i}$ and $R_{j}$ of distance $d$ as $\delta=R_{i}+R_{j}-d$; interactions vanish for $\delta<0$. The normal force between two particles consists of repulsive and attractive contributions. The repulsive part (Pöschel \& Schwager 2005),

$f_{\text {rep }}=\frac{4}{3} M^{*} \sqrt{R^{*} \delta}\left(\delta+A v_{\mathrm{n}}\right)$,

consists of a Hertzian $\delta^{3 / 2}$ contribution, based on elastic theory, and a dissipative part, characterizing a viscoelastic contact (Brilliantov et al. 1996). Here $R^{*}=R_{i} R_{j} /\left(R_{i}+R_{j}\right)$ is the reduced radius for a collision of two particles with radii $R_{i}$ and $R_{j}, M^{*}=Y /\left[2\left(1-v^{2}\right)\right]$ is the reduced modulus, $Y$ Young's modulus, $v$ Poisson's ratio, $v_{\mathrm{n}}$ is the velocity component in normal direction, and $A$ is an empirical constant modeling dissipation. The attractive part of the normal force is proportional to the specific surface energy $\gamma$ as (Derjaguin et al. 1975; Maugis 2000; Blum 2006)

$f_{\text {adh }}=8 \pi R^{*} \gamma$.

The tangential forces between two grains comprise several friction forces. The gliding friction,

$f_{\text {slide }}=\frac{1}{2} G \pi a^{2}$,

depends on the shear modulus $G=Y /[2(1+v)]$ and the radius $a=$ $\sqrt{\delta R^{*}}$ of the contact area (Burnham \& Kulik 1999). The bond forming and breaking process during rolling motion leads to energy dissipation and a decelerating torque (Dominik \& Tielens 1997),

$D_{r}=2 f_{\text {adh }} \xi_{\text {yield }}$.
Here, $\xi_{\text {yield }}$ is the distance for which two grains can roll over each other without breaking their atomic contacts. Finally, torsional friction leads to a decelerating torque (Dominik \& Tielens 1997)

$D_{t}=\frac{1}{3} G \frac{a^{3}}{\pi}$.

Further details on the derivation of the forces are given by Ringl \& Urbassek (2012).

In our simulations we use the materials parameters for silica, $Y=54 \mathrm{GPa}, v=0.17$, hence $M^{*}=27.8 \mathrm{GPa}$, and $\gamma=25 \mathrm{~mJ} \mathrm{~cm}^{-2}$ (Chokshi et al. 1993). The dissipation constant $A=0.5 \mathrm{~ns}$ is chosen such that the restitution coefficient for a collision of two grains with velocity $v=1 \mathrm{~m} \mathrm{~s}^{-1}$ amounts to $\epsilon=0.69$ (Poppe et al. 2000; Ringl \& Urbassek 2012). The parameter describing rolling friction, $\xi_{\text {yield }}$, is taken to be $1 \AA$ (Dominik \& Tielens 1997).

Summarizing, the difference in the collision behavior of the central chondrule and the dust grain enters in this granularmechanics approach by the different radii of these two particles. Via the formulae given above, the radius enters all the intergranular forces: repulsive and attractive interaction as well as gliding, rolling and torsional friction. All materials specific properties, on the other hand, are identical for both grains and chondrule.

\section{Results}

The central collision of two naked chondrules leads to a bouncing velocity of $2.4 \mathrm{~mm} \mathrm{~s}^{-1}$; below this velocity two chondrules stick as the collision energy is dissipated (term proportional to $A$ in Eq. (1)) and the adhesion, Eq. (2), then prevents the two chondrules from separating again. As a comparison, the collision of two dust grains has a bouncing velocity of $44 \mathrm{~cm} \mathrm{~s}^{-1}$ in our algorithm; the experiment (Poppe et al. 2000) gives values of around $1 \mathrm{~m} \mathrm{~s}^{-1}$.

Krijt et al. (2013) - based on previous work (Johnson 1985; Chokshi et al. 1993; Dominik \& Tielens 1997; Thornton \& Ning 1998) - provide an analytical formula for the bouncing velocity as

$v_{\mathrm{b}}=\left(\frac{14.18}{m^{*}}\right)^{1 / 2}\left(\frac{\gamma^{5} R^{* 4}}{M^{* 2}}\right)^{1 / 6}$,

where $m^{*}=m / 2$ and $m$ is the mass of a particle. For a collision between two identical spheres, this can be simplified as

$v_{\mathrm{b}}=\frac{1.639}{\rho^{1 / 2}}\left(\frac{\gamma^{5}}{M^{* 2} R^{5}}\right)^{1 / 6}$.

Evaluation for our material parameters gives $v_{\mathrm{b}}=3.83$ (70.3) $\mathrm{mm} \mathrm{s}^{-1}$ for the chondrule (grain) collision. According to Eq. (6), the bouncing velocity for the asymmetric collision between a grain and a chondrule agrees within a factor of $2^{1 / 6}=$ 1.12 to that between two grains; this feature is also reproduced in the simulations.

The exact numbers obtained from Eq. (7) differ from our simulation results for two reasons. First, Eq. (6) assumes the Johnson-Kendall-Roberts (JKR) theory (Johnson et al. 1971) to describe the adhesive contact, while our formula (2) is inspired by the Derjaguin-Muller-Toporov (DMT) theory (Derjaguin et al. 1975). The DMT theory is assumed to describe stiff and small spheres well, while JKR is more applicable to compliant large spheres (Greenwood 1997; Maugis 2000). Second, Eq. (6) applies to an elastic adhesive collision 


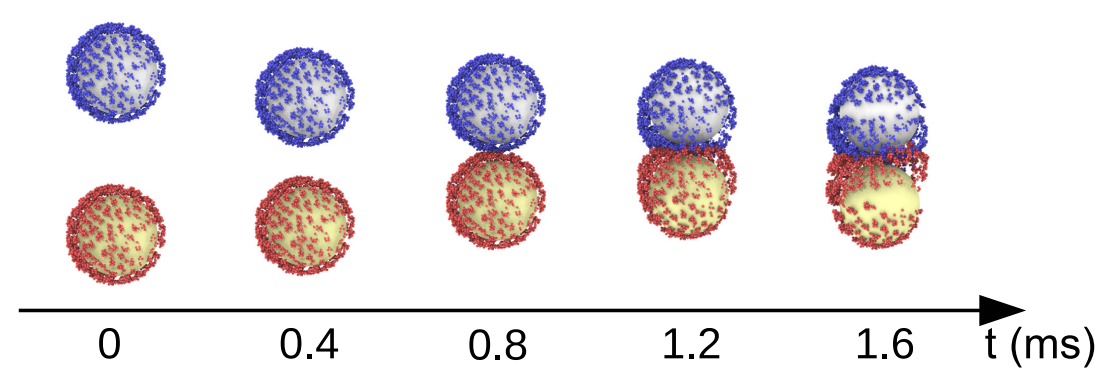

(a)

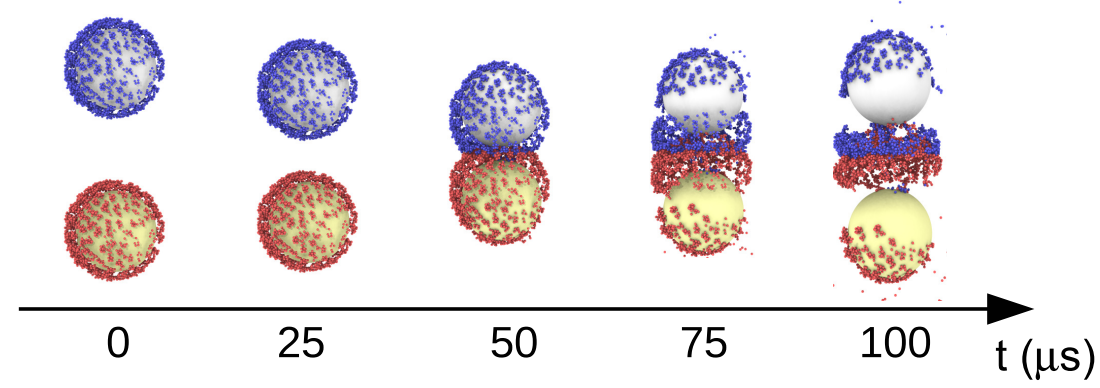

(b)

Fig. 1. Snapshots of two chondrules covered with a dust shell of thickness $d=5 \mu \mathrm{m}$ and filling factor $\phi=0.15$ colliding with a velocity of a) 0.05 and b) $0.75 \mathrm{~m} \mathrm{~s}^{-1}$. Color differentiates the two chondrules to which a grain initially belonged. Times are indicated on the horizontal axis.

(Krijt et al. 2013), while we include energy dissipation during the collision by the viscoelastic dissipation constant $A$ in Eq. (1). However, the basic $R^{-5 / 6}$ dependence of the bouncing velocity on the particle radius is fulfilled well in our simulations.

Figure 1 exemplifies the collision of two chondrules covered with a dust shell of thickness $d=5 \mu \mathrm{m}$ and filling factor $\phi=0.15$ for the two different collision velocities 0.05 and $0.75 \mathrm{~m} \mathrm{~s}^{-1}$. The collision at the lower velocity, Fig. 1a, is clearly nonbouncing; the dust shell around the two chondrules dissipates the collision energy and leads to cohesion of the final aggregate. There is a strong movement of the dust shell toward the contact area of the two chondrules. The concentration of the grains in this region helps in dissipating the kinetic energy and glues the two chondrules together.

At higher collision energy, Fig. 1b, the chondrules separate after the collision. Here an even stronger reorganization of the dust shells upon collision has occurred. In particular the chondrule hemispheres neighboring the collision area were emptied of their dust shells. The dust grains have assembled in between the two separating chondrules; while they are still in loose contact with one of the chondrules, they lost contact with the other chondrule entirely. Several grains were also ejected by the collision as monomers (i.e., sputtered).

Porous aggregates, with similar size and porosities as the dust shells used here, always stick and never bounce off each other in our simulations (Gunkelmann et al. 2016); for higher velocities $\left(v>20-30 \mathrm{~m} \mathrm{~s}^{-1}\right)$ the merged aggregate starts fragmenting. Wada et al. (2011) and later Seizinger \& Kley (2013) find in related simulation work that bouncing only occurs for denser aggregates with filling factors $\phi \geq 0.4$; this feature is supported by experiment (Kothe et al. 2013). This fact emphasizes how the chondrules inside our aggregates totally change the collisional outcome of dust aggregates.

We calculate the relative velocity, $\left|\boldsymbol{v}_{1}-\boldsymbol{v}_{2}\right|$, of the two chondrules after the collision; the coefficient of restitution is then obtained as

$\epsilon=\frac{\left|\boldsymbol{v}_{1}-\boldsymbol{v}_{2}\right|}{v}$.

In Fig. 2 this coefficient is shown as a function of the collision velocity, $v$, for various densities and thicknesses of the dust shell. The data are averaged over three individual collision events in which the initial chondrule orientations were randomly rotated. As long as $\left|\boldsymbol{v}_{1}-\boldsymbol{v}_{2}\right|=0$, the chondrules stick. The figure verifies that sticking occurs for velocities far above the bouncing velocity of naked chondrules. For the thickest shell, $d=5 \mu \mathrm{m}$, the bouncing velocities are between $0.25 \mathrm{~m} \mathrm{~s}^{-1}$ (for $\phi=0.08$ ) and $0.75 \mathrm{~m} \mathrm{~s}^{-1}$ (for $\phi=0.21$ ). Even after bouncing occurs, the restitution coefficient is far below 1 ; this demonstrates a strong damping effect of the dust shells. Figure 2a quantifies that dense dust shells lead to a higher bouncing velocity than dust shells of low filling factor and Fig. $2 \mathrm{~b}$ demonstrates to which extent thick dust shells are more efficient in modifying the collision than thin shells. We note here that the thinnest shells, $d=1.5$ and $2 \mu \mathrm{m}$, have only a small effect on the collision, as the restitution coefficient is close to 1 , in particular, for the thinnest shell. This appears plausible in view of the fact that individual dust grains have diameters of $1.52 \mu \mathrm{m}$; for a filling factor of only 0.081 , hence, no or only very few dust grains interact with the chondrules in the collision area. The thicker shells, $d=3$ and $5 \mu \mathrm{m}$, however, have a clearly visible effect on the collision outcome.

Finally, in Fig. 3 we show the effect of the collision on the dust grains by showing the fraction of the grains that are still in contact with one of the chondrules after the collision. As shown in Fig. 1, at higher speeds a sizable part of the grains may be sputtered as monomers or may aggregate to form a dust agglomerate, which (partly) loses contact with the chondrules. The figure demonstrates that this effect is indeed continuous in that it increases constantly with collision velocity. No sharp jumps are discernible at the bouncing velocity, but also grains are ejected from the dust shells below this velocity. This figure demonstrates 


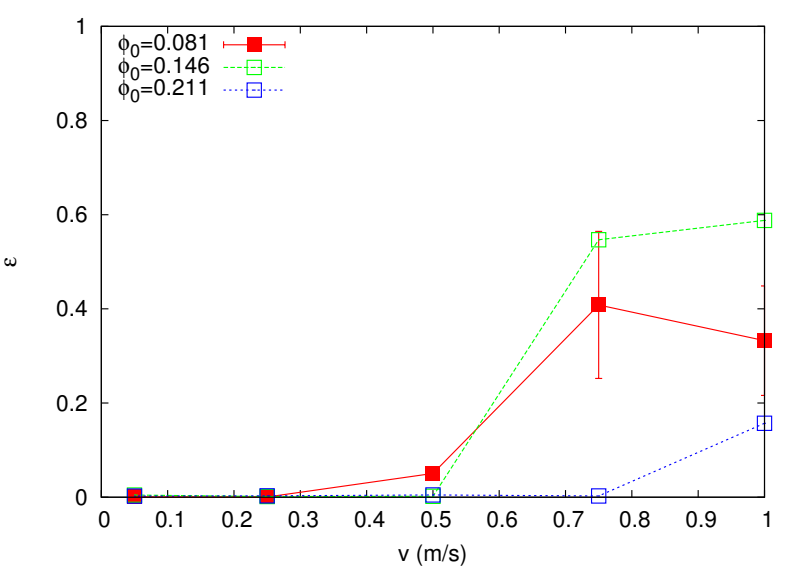

(a)

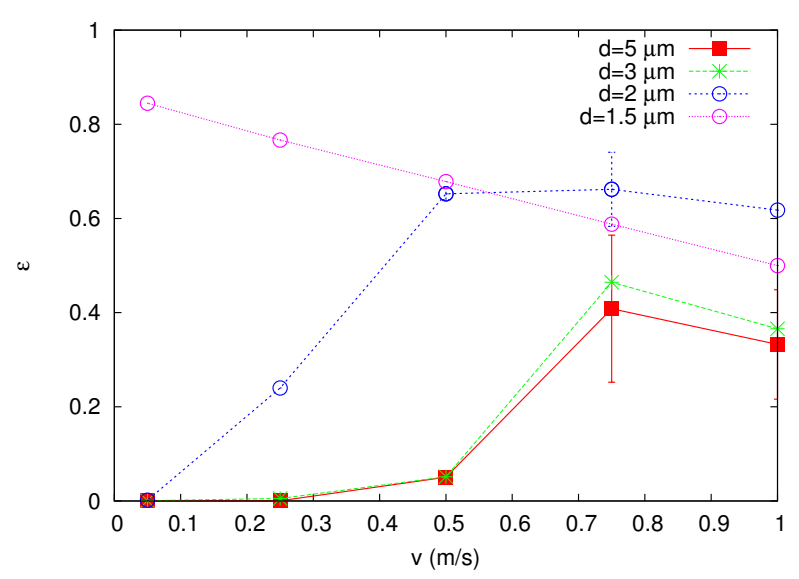

(b)

Fig. 2. Dependence of the coefficient of restitution, $\epsilon$, of two collided chondrules on their collision velocity $v$. The chondrules are covered a) with a dust shell of fixed thickness $d=5 \mu \mathrm{m}$ and varying filling factor $\phi$, and $\mathbf{b}$ ) with a dust shell of fixed filling factor $\phi=0.081$ and varying thickness $d$. A representative error is included.

that while the dust shell helps in accommodating the collision energy of the chondrules, it becomes destroyed by this process even if the two chondrules stick after the collision.

\section{Summary}

Using granular-mechanics simulations we investigated the influence of a dust shell on the collision behavior of two chondrules. For the specific cases shown, the bouncing velocity of the two chondrules increases by two orders of magnitude, if a dust shell covers the chondrules, from $2.4 \mathrm{~mm} \mathrm{~s}^{-1}$ to $0.75 \mathrm{~m} \mathrm{~s}^{-1}$ for the densest and thickest shell studied. The shells become partly destroyed during the collision process, both by sputtering (monomer ejection) and by agglomeration to dust aggregates. Thicker and denser dust shells are more efficient in accommodating the collision energy than thin and porous shells.

Acknowledgements. Simulations were performed at the High Performance Cluster Elwetritsch (RHRK, TU Kaiserslautern, Germany). A.K. acknowledges support by a JSPS Fellowship.

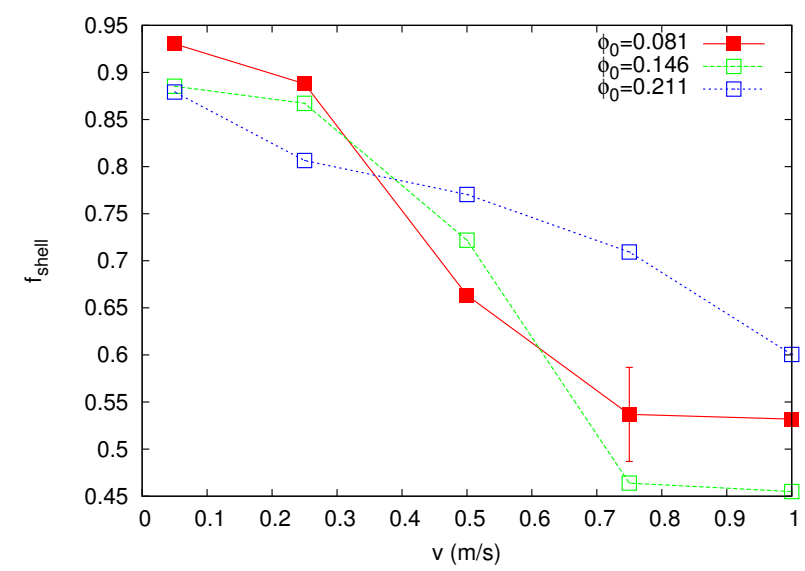

Fig. 3. Dependence of the fraction of the dust shell, $f_{\text {shell }}$, that covers the chondrules after collision on the collision velocity $v$. The chondrules are covered with a dust shell of fixed thickness $d=5 \mu \mathrm{m}$ and varying filling factor $\phi$. A representative error is included.

\section{References}

Beitz, E., Güttler, C., Weidling, R., \& Blum, J. 2012, Icarus, 218, 701 Birnstiel, T., Fang, M., \& Johansen, A. 2016, Space Sci. Rev., 205, 41 Blum, J. 2006, Adv. Phys., 55, 881

Blum, J. 2010, Res. Astron. Astrophys., 10, 1199

Blum, J., \& Schräpler, R. 2004, Phys. Rev. Lett., 93, 115503

Brilliantov, N. V., Spahn, F., Hertzsch, J.-M., \& Pöschel, T. 1996, Phys. Rev. E, 53,5382

Burnham, N., \& Kulik, A. A. 1999, in Handbook of Micro/Nano Tribology, 2nd edn., ed. B. Bhushan (Boca Raton: CRC Press), 247

Chokshi, A., Tielens, A. G. G. M., \& Hollenbach, D. 1993, ApJ, 407, 806

Cuzzi, J. N. 2004, Icarus, 168, 484

Derjaguin, B. V., Muller, V. M., \& Toporov, Y. P. 1975, J. Colloid Interface Sci., 53, 314

Dominik, C., \& Tielens, A. G. G. M. 1997, ApJ, 480, 647

Greenwood, J. A. 1997, Proc. Roy. Soc. London A, 453, 1277

Grün, E. 2007, in Encyclopedia of the solar system, 2nd edn., eds. L. A. McFadden, P. R. Weissman, \& T. V. Johnson (New York: Academic Press), 621

Gunkelmann, N., Ringl, C., \& Urbassek, H. M. 2016, A\&A, 589, A30

Güttler, C., Blum, J., Zsom, A., Ormel, C. W., \& Dullemond, C. P. 2010, A\&A, 513, A56

Johnson, K. L. 1985, Contact mechanics (Cambridge: Cambridge University Press)

Johnson, K. L., Kendall, K., \& Roberts, A. D. 1971, Proc. R. Soc. London Ser. A, 324, 301

Kloss, C., Goniva, C., Hager, A., Amberger, S., \& Pirker, S. 2012, Prog. Comput. Fluid Dyn., 12, 140

Kothe, S., Blum, J., Weidling, R., \& Güttler, C. 2013, Icarus, 225, 75

Krijt, S., Güttler, C., Heis̈selmann, D., Dominik, C., \& Tielens, A. G. G. M. 2013, J. Phys. D, 46, 435303

Maugis, D. 2000, Contact, adhesion and rupture of elastic solids (Berlin: Springer)

Metzler, K., Bischoff, A., \& Stöffler, D. 1992, Geochim. Cosmochim. Acta, 56, 2873

Ormel, C. W., Cuzzi, J. N., \& Tielens, A. G. G. M. 2008, ApJ, 679, 1588

Poppe, T., Blum, J., \& Henning, T. 2000, ApJ, 533, 454

Pöschel, T., \& Schwager, T. 2005, Computational granular dynamics: models and algorithms (Springer)

Ringl, C., \& Urbassek, H. M. 2012, Comput. Phys. Commun., 183, 986

Ringl, C., \& Urbassek, H. M. 2013, Comput. Phys. Commun., 184, 1683

Seizinger, A., \& Kley, W. 2013, A\&A, 551, A65

Thornton, C., \& Ning, Z. 1998, Powder Technol., 99, 154

Wada, K., Tanaka, H., Suyama, T., Kimura, H., \& Yamamoto, T. 2007, ApJ, 661, 320

Wada, K., Tanaka, H., Suyama, T., Kimura, H., \& Yamamoto, T. 2011, ApJ, 737, 36

Weidling, R., Güttler, C., \& Blum, J. 2012, Icarus, 218, 688

Zsom, A., Ormel, C. W., Güttler, C., Blum, J., \& Dullemond, C. P. 2010, A\&A, 513, A57 